\title{
lavadero móvil para áridos
}

S I N $\mathbf{N}$ O

La conocida empresa Eagle Iron Works, de Des Moines, Iowa (EE. UU.), ha perfeccionado una serie de modelos de lavaderos móviles para la depuración de áridos, que, junto con otros dispositivos anxiliares, de la misma casa, constituyen instalaciones de clasificación, lavado y desecación de áridos de las granulometrías ordinarias empleadas en la preparación de hormigones. En este trabajo se describen estas instalaciones.

\section{Generalidades}

La calidad de los áridos destinados a la fabricación del hormigón es un factor determinante de las características físicas y mecánicas de un hormigón bueno. Además, la descomposición del hormigón en el tiempo, debido a la introducción de agentes nocivos que pudieran acompañar a los áridos, adquiere una gran importancia en la estabilidad y resistencia del material; es más, hay veces que la velocidad de reacción de los distintos procesos químicos inherentes a todo fraguado de la papilla formada por el aglomerante al mezclarse con el agua, se ve netamente alterada debido a la presencia del propio árido y las substancias que por mil causas distintas le pudieran acompanar.

Aunque químicamente se podría llegar a la determinación de materias capaces de contrarrestar la acción nociva de estos cuerpos extraños en los áridos, fenómeno que no debe olvidarse al tratar de elegir un árido, el proceso o tratamiento actualmente mas viable es el mecanico, es decir, lavado, decantacion y sedimentacion gravimétrica, ya que, de momento, los mejores bancos explotables de canteras o graveras naturales de mina o río dan materiales de composición química de marcada irregularidad: menor en las canteras que explotan bancos potentes sanos muy uniformes que en las graveras.

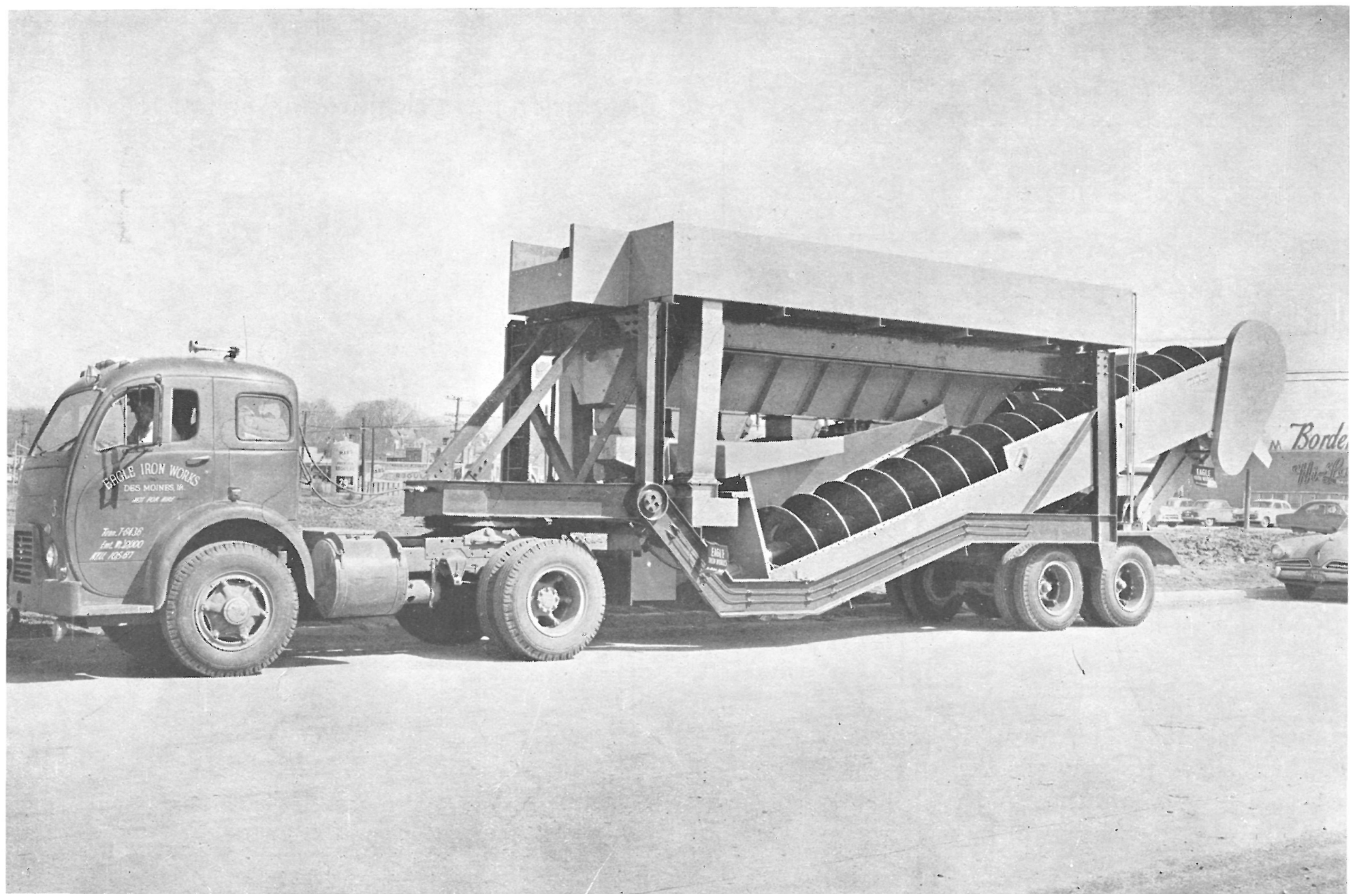


esquema de instalación general

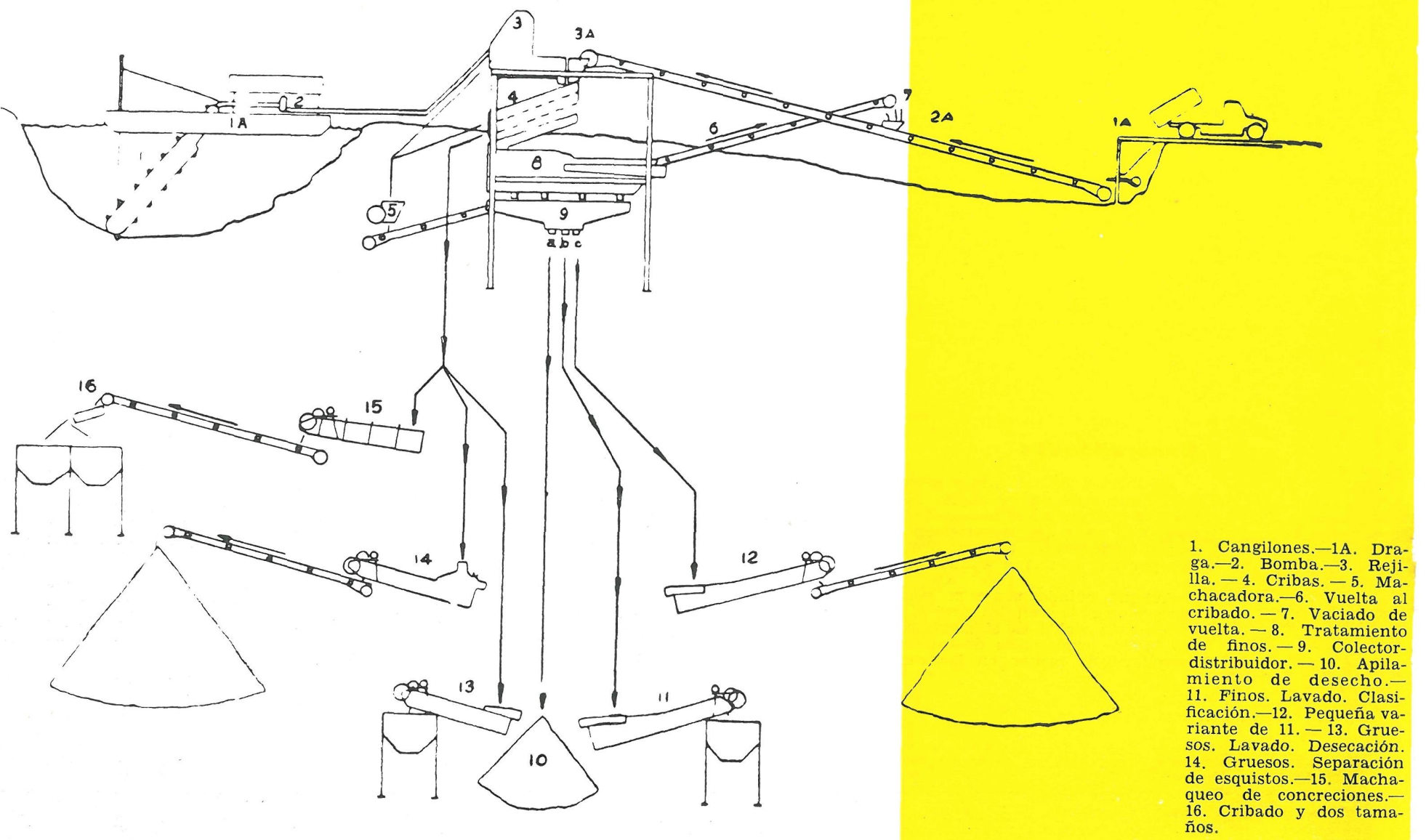

instalación para clasificación en tres tamaños

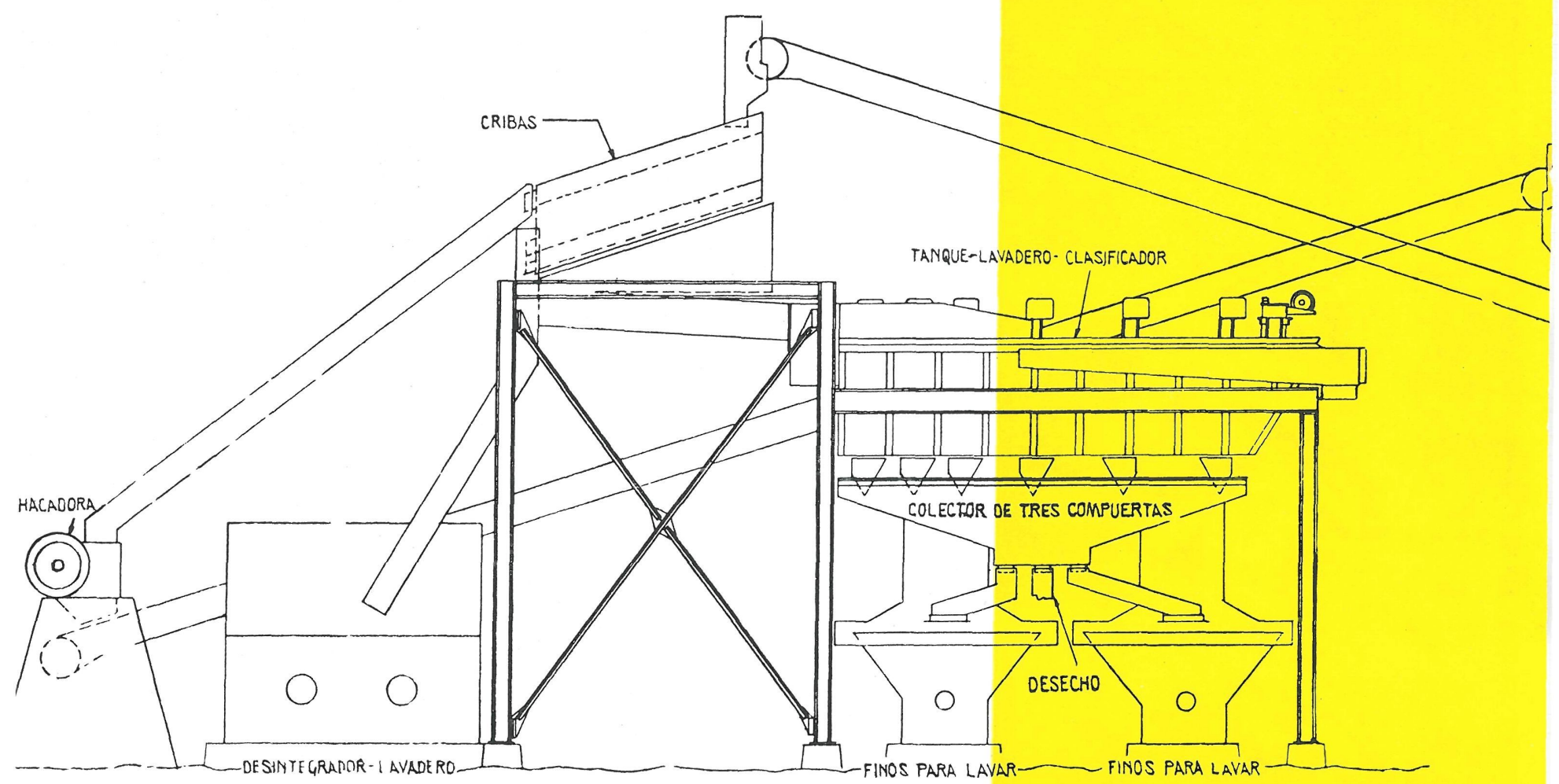




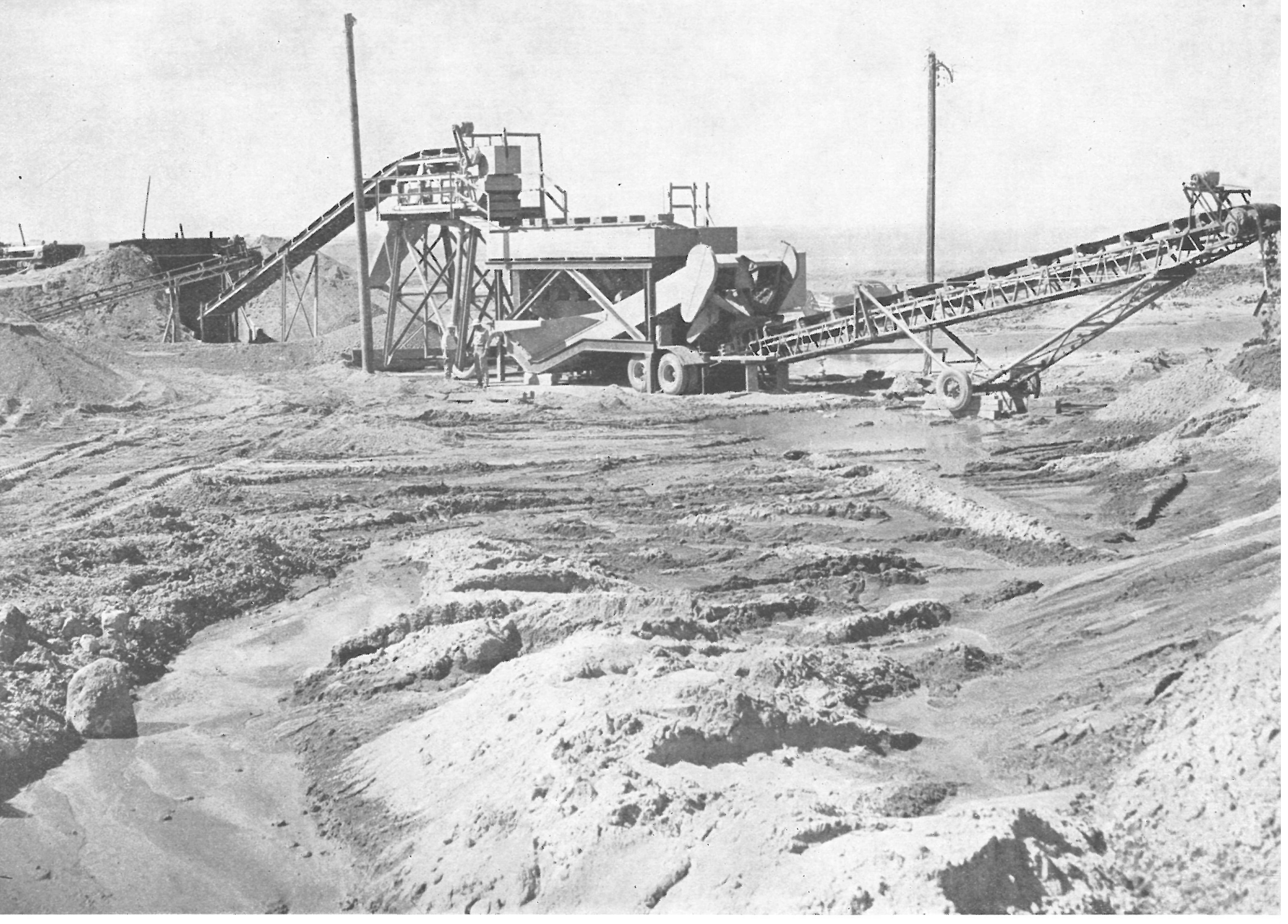

En las graveras de ríos, torrenteras y de capas poco profundas se corre el riesgo de arrastrar una gran acumulación de materias orgánicas durante el arranque.

Hay casos ciertamente curiosos en los que el hormigón no experimenta el propio proceso de endurecimiento, caso excepcional y grave; pero, en otro orden de importancia y magnitud, el hormigón presenta una gran irregularidad de sus cualidades mecanicas debido a los áridos y agua, ya que estos materiales son siempre más irregulares que los aglomerantes, cuyo proceso de fabricación se cuida escrupulosamente.

De todos los procedimientos actuales de que disponemos para la depuración de áridos, el lavado ordinario con agua es el mejor, más económico y de sencilla realización. Claro está, presenta el inconveniente, algunas veces, de que el agua escasea; no obstante esto, el lavado de áridos es la mejor purificación en general.

\section{El lavado portátil}

El lavadero portátil consiste, en esencia, de una o dos hélices continuas (espirales de Arquímedes), que recogen los áridos en su pie $\mathrm{y}$, animándolas de un movimiento de rotación, impulsan el material hacia arriba en un canalón que envuelve para presión, y por medio de unos dispositivos de cabeza se pueden eliminar ciertos materiales que ensucian los áridos.

Este dispositivo tan sencillo sirve también de secadero cuando el flujo de alimentación, como el que podría provenir de un dragado de materiales de fondo, llega al pie de la espiral mezclado con agua. 

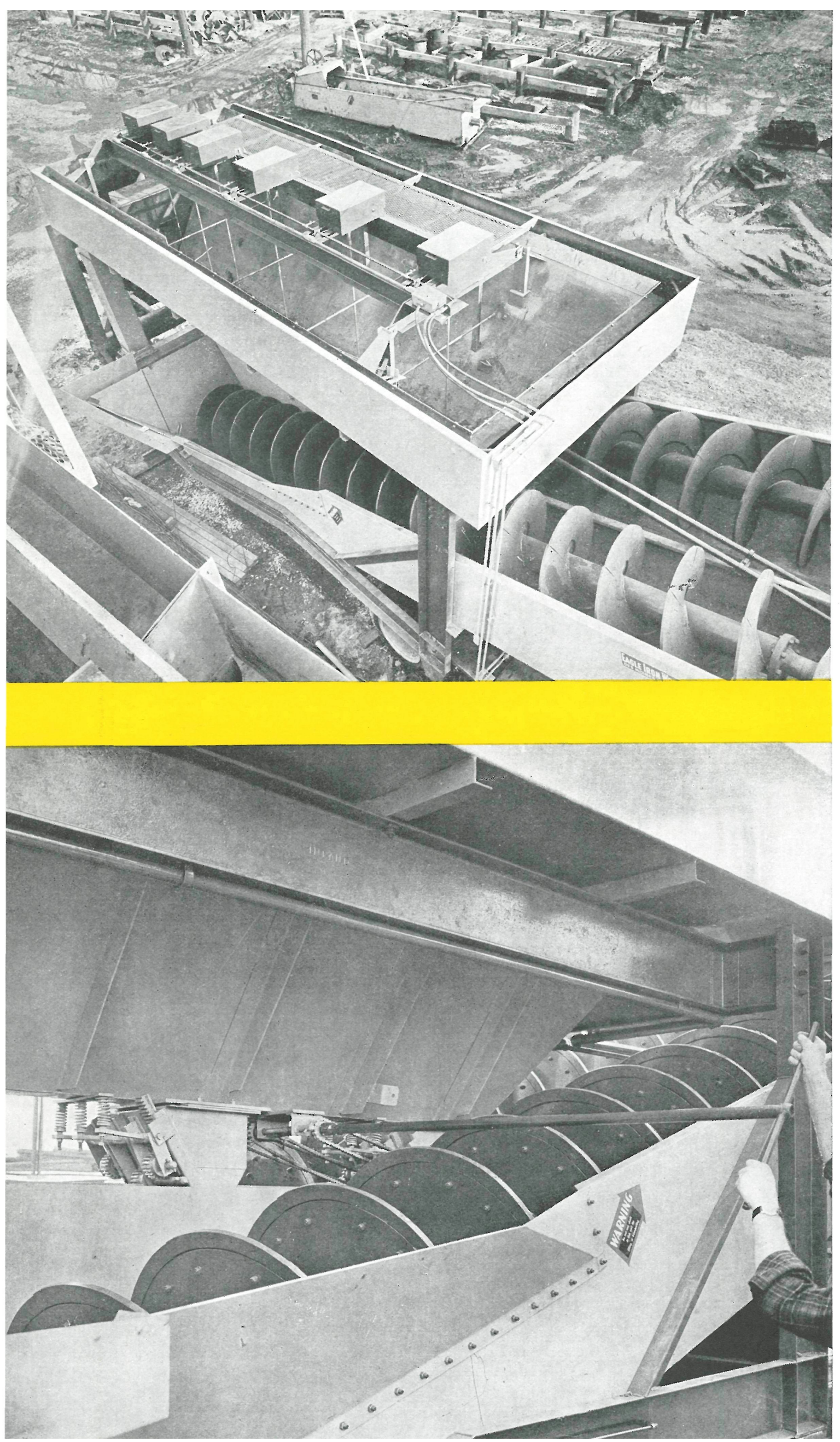

Instalación para la cla. sificación en tamaños.

Reajuste de las compuertas del tanque clasifica. 


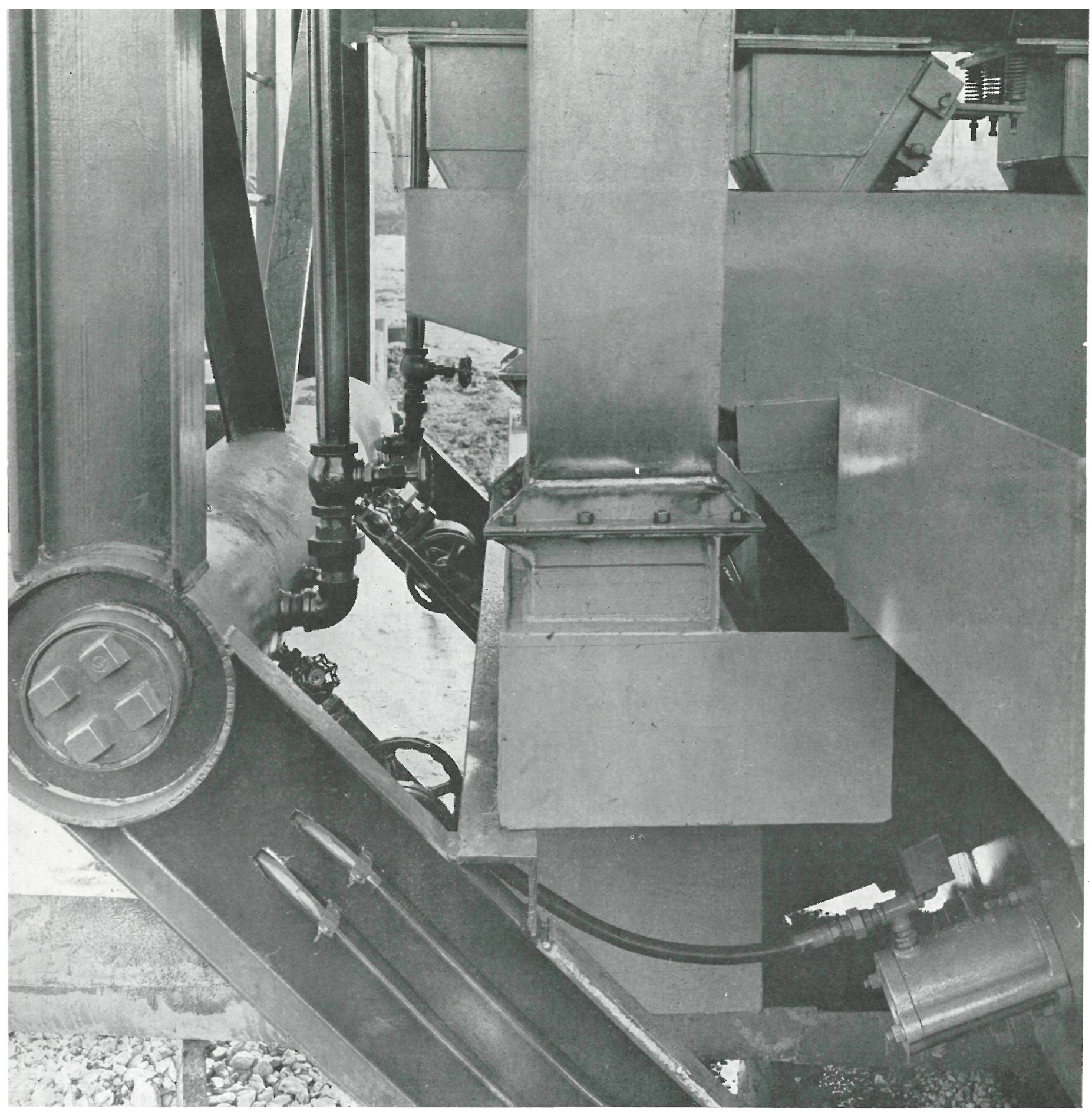

A este tipo de lavadero le complementan dos tanques de lavado y clasificación, de los cuales, por medio de compuertas de fondo, se pueden establecer separaciones por tamaños.

Como los áridos pueden presentarse formando concreciones más o menos apelmazadas y tenaces, la Eagle Iron Works ha previsto esta emergencia, que salva con un dispositivo similar en principio a las espirales, pero que sustituye a éstas por una serie de tocones robustos capaces de bracear los áridos enérgicamente y hacerles sufrir un machaqueo.

Los movimientos de áridos de una a otra de estas máquinas en el proceso del tratamiento se realiza siempre con cintas transbordadoras, procedimiento que también se emplea para el apilamiento de materiales tratados.

\section{Dimensiones y características principales de las espirales}

Como se dijo anteriormente, existen varios tipos de estas máquinas, que, aunque en el fondo se basan todas en el mismo principio, sus dimensiones, agrupación y rendimierto varían de unas a otras.

Las espirales simples tienen diámetros que varían de 0,50 a 0,60 . Su longitud total es también variable y va de 6,70 a $7,60 \mathrm{~m}$. La velocidad varía de 38 a 32 revoluciones por miliuto; el rendimiento es de 30 a 50 toneladas por hora; el agua necesaria es de 100 a 900 litros por minuto, suministrados a $1,75 \mathrm{~kg} / \mathrm{cm}^{3}$ de presión.

La dimensión máxima de los materiales que se han de tratar en este tipo particular es de unos 10 mm; y la potencia, de acuerdo con los rendimientos, varía de 5 a 10 caballos. 


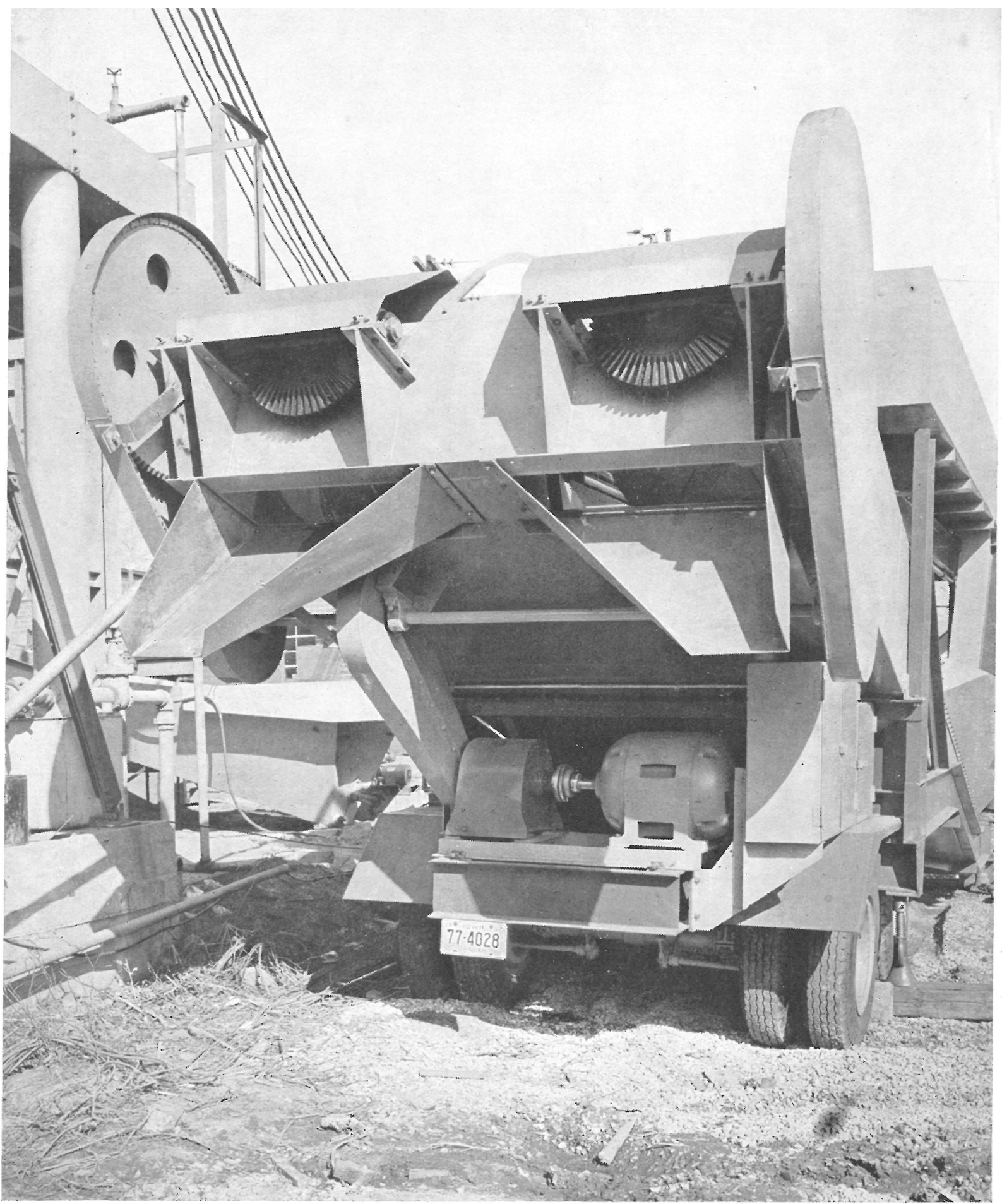

Fotos: UNIVERSAL

En los modelos de espirales gemelos, empleados en las casas que se requiere un gran rendimiento, los diámetros de las espiras son de 0,90 a $1,40 \mathrm{~m}$; la longitud, de 6,70 a $10,40 \mathrm{~m}$; la capacidad, de hasta 500 toneladas por hora; de 25 a 75 revoluciones por minuto; necesitan hasta $4 \mathrm{~m}^{3}$ de agua por minuto, y una potencia de 75 caballos. Las dimensiones máximas de los materiales que se pueden tratar es de urıos 10 milímetros.

\section{Instalaciones}

Con objeto de dar una idea general de las instalaciones que se pueden hacer con estos lavaderos móviles, tomaremos como base dos tipos de instalación: uno amplio, en el que se puede apreciar la disposición de los distintos elementos, y otro para obtener tres clases de tamaños.

En el primero, representado en el esquema adjunto, la instalación es muy detallada y supone existe una doble alimentación, ya por draga o por cantera, o de las dos simultaneamente. También se ha previsto el machaqueo. El esquema es tan sencillo que no requiere explicación para su comprensión.

El segundo tipo de instalación, para tres tamaños, contando igualmente con machaqueo, representado con más detalles en la figura adjunta, no presenta dificultad alguna de montaje y explotación.

J. J. U. 